\title{
Decomposition Reaction of Organophosphorus Nerve Agents on Solid Surfaces with Atmospheric rf Plasma Generated Gaseous Species
}

\author{
Seong H. Kim, ${ }^{1, *}$ Jeong Hoon Kim, ${ }^{2}$ and Bang-Kwon Kang ${ }^{3}$
}

Figure S-1. Photographs of Drosophila enclosed in culture bottles containing parathion and paraoxon samples treated with different methods. The tested OP molecule and treatment condition are given in each picture. The UV and UV/ozone treated sample pictures were taken after 12 hour culture. The plasma treated sample pictures were taken after 4 week culture.

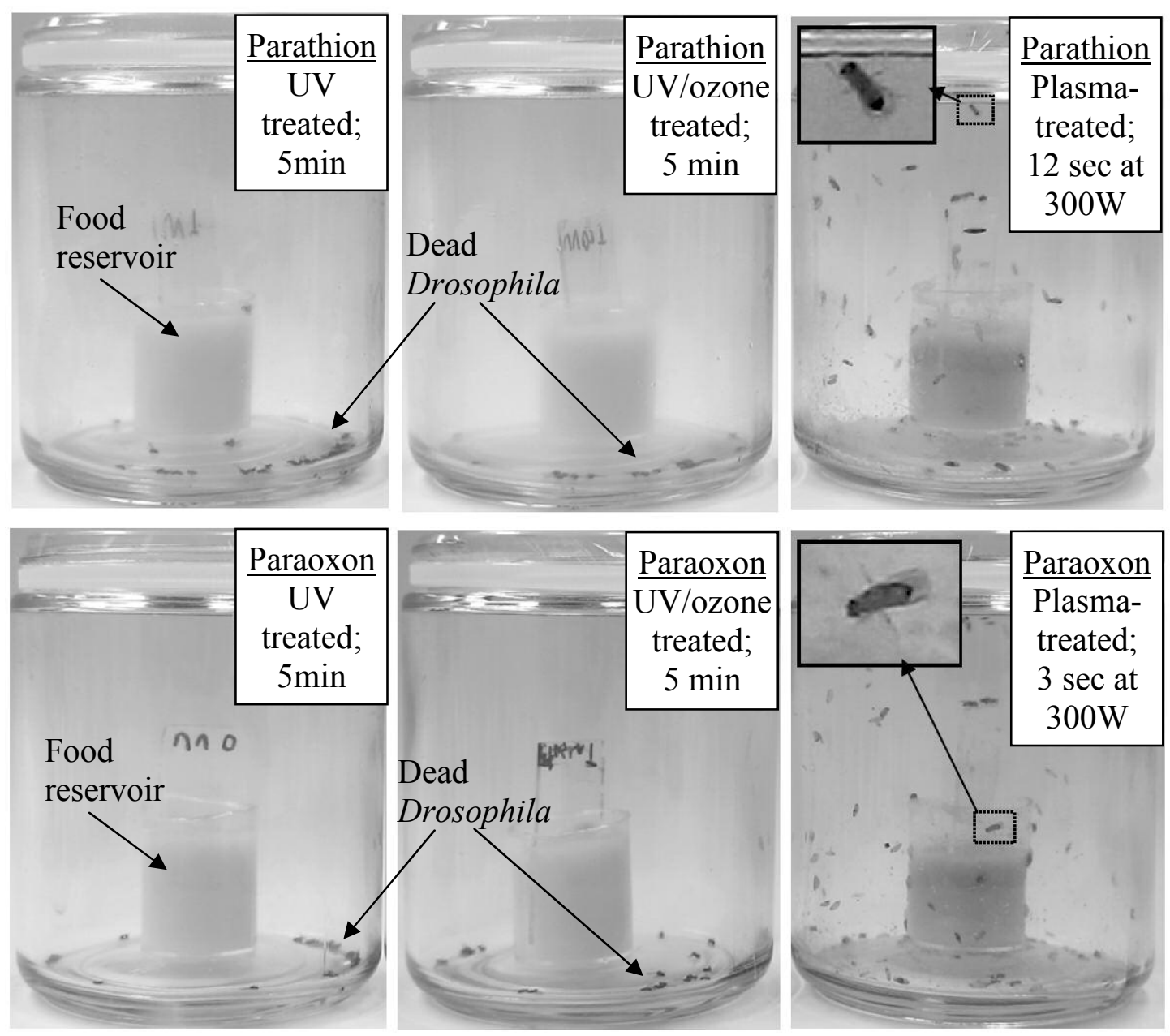

